\title{
Colorectal Carcinoma: Diagnostic, Prognostic, and Molecular Features
}

\author{
Carolyn C. Compton, M.D., Ph.D. \\ Department of Pathology and University Health Center, McGill University, Montreal, Quebec
}

\begin{abstract}
Surgical resection is the primary treatment modality for colorectal cancer, and the pathologic assessment of the resection specimen provides data that is essential for patient management, including the estimation of postoperative outcome and the rationale for adjuvant therapy. The essential elements of the pathological assessment of colorectal cancer resection specimens include the pathologic determination of TNM stage, tumor type, histologic grade, status of resection margins, and vascular invasion. The prognostic and/or predictive value of these elements, as well as guidelines for their derivation and interpretation, are reviewed in detail. Other tissue-based prognostic factors that are strongly suggested by existing data to have stageindependent prognostic value or to predict response to adjuvant therapy but that have not yet been validated for routine patient care are also reviewed. These include perineural invasion, tumor border configuration, host immune response to tumor, and molecular features such as microsatellite instability or loss of heterozygosity of chromosome 18. The need for high-quality, reproducible pathologic data in the care of the colorectal cancer patient, and the dependence of that data on standardization of all aspects of pathological assessment, is emphasized.
\end{abstract}

KEY WORDS: Circumferential margin, Colon cancer, Grade, Microsatellite instability, Pathology, Prognostic factors, Rectal cancer, TNM stage.

Mod Pathol 2003;16(4):376-388

Copyright ( $\odot 2003$ by The United States and Canadian Academy of Pathology, Inc.

VOL. 16, NO. 4, P. 376, 2003 Printed in the U.S.A.

Date of acceptance: January 8, 2003.

Address reprint requests to: Carolyn C. Compton, M.D., Ph.D., Department

of Pathology, McGill University, 3775 University Street, Montreal, Quebec

H3A 2B4, Canada; fax: 514-398-7446; e-mail: compton@med.mcgill.ca.

DOI: 10.1097/01.MP.0000062859.46942.93
Colorectal cancer is second only to lung cancer as a cause of cancer-related death in North America. More than 130,000 new cases of colon and rectal cancer were reported in 2000 in the United States. In short, colorectal cancer is by far the most common malignancy of the gastrointestinal tract, and it is, without question, a "surgical disease." An estimated $92 \%$ of colon cancer patients and $84 \%$ of rectal cancer patients undergo surgical resection as the primary modality of treatment; the procedure is most typically performed with curative intent. The appropriateness of adjuvant therapy and the prediction of outcome for the patient are, to a large extent, based on the pathologic assessment of the local disease and other tissue-based prognostic factors in the resection specimen. The problems and controversies associated with the assessment of the colorectal resection specimen, and the current recommendations for their interpretation and reporting, are reviewed.

\section{SPORADIC COLORECTAL CANCER: THE BASICS THAT ARE NOT SO BASIC}

The pathology report of a colorectal cancer resection specimen typically documents the anatomic site of the malignancy, the histologic type, the parameters that determine the local tumor stage, and the histopathologic confirmation of distant metastasis, if applicable. Other reported features include those having additional prognostic (related to likelihood of survival) or predictive (related to likelihood of response to therapy) value, as well as those that may be important for clinicopathologic correlation or quality control (e.g., actual tumor size versus size measurement by imaging techniques). Although basic to pathologic assessment, the definition and interpretation of many of these key features is controversial. The lack of consensus on and standardization of interpretation of these features is the basis of much of the variation in pathologic assessment of colorectal cancers. Variability in assessment, in turn, compromises the quality of pathologic data vital to patient management and clinical research. The major bones of contention and sources of variation are discussed individually. 
The Anatomic Site of the Tumor: Where Is the Rectum? The Raging Debate

The anatomic site of a tumor is important for three reasons:

1. It determines the appropriate staging system (further discussed later in the article).

2. It defines the lymph nodes that are regional (assigned to the $\mathrm{N}$ category of tumor, nodes, metastasis [TNM] staging) versus those that are nonregional (assigned to the $\mathrm{M}$ category).

3. It determines whether or not a circumferential (nonperitonealized radial) margin is relevant.

Anatomic site is often documented by measurement from known landmarks according to general guidelines defining colonic topography. In general, four major anatomic divisions of the colon are recognized: the right (ascending) colon, the middle (transverse) colon, the left (descending) colon, and the sigmoid colon (1). The right colon is subdivided into the cecum (peritoneally located and measuring about $6 \times 9 \mathrm{~cm}$ ) and the ascending colon (retroperitoneally located and measuring 15 to $20 \mathrm{~cm}$ long). The descending colon, also located retroperitoneally, is 10 to $15 \mathrm{~cm}$ in length. The descending colon becomes the sigmoid colon at the origin of the mesosigmoid, and the sigmoid colon becomes the rectum at the termination of the mesosigmoid. The proximal aspect of the rectum is covered by peritoneum on the anterior surface only. The distal aspect has no peritoneal covering.

Exactly where the rectum ends and the anal canal begins, however, is a highly controversial and critically important issue. This is not a trivial matter because adenocarcinomas of the rectum and anal canal are staged completely differently. The $\mathrm{T}$ category of colorectal cancer is defined by extent through the wall, whereas the $\mathrm{T}$ category (i.e., $\mathrm{T} 1$ and T2) of anal canal cancer is defined by tumor size. The $\mathrm{N}$ category of colorectal cancers is defined by number of affected nodes, whereas the $\mathrm{N}$ category for anal cancers is defined by the location of affected nodes.

The 5th edition of the staging manual of the American Joint Committee on Cancer (AJCC) provided two conflicting descriptions of the rectum (1). On the one hand, it defined the rectum as the "distal $1 \mathrm{~cm}$ of large intestine as measured from the anal verge with a sigmoidoscope" [emphasis added], but then went on to say that the rectum is "approximately $12 \mathrm{~cm}$ in length" [emphasis added]. More recently, in the "Guidelines for Colon and Rectal Cancer Surgery" (2), the rectum was defined by consensus as being $\leq 12 \mathrm{~cm}$ from the anal verge by rigid proctoscopy. This definition was considered justifiable on a biologic basis because clinical observations indicate that the patterns of recurrence of tumors $>12 \mathrm{~cm}$ are more consistent with colonic cancers than rectal cancers. This definition of the proximal border of the rectum now is included in the 6th edition of the AJCC staging manual (3). Unfortunately, by either the previous or the revised definition, the dividing line between rectum and anal canal is not an anatomic landmark for pathologists. If it is not clear where the rectum begins, it is even less certain where it ends, because its length is ill-defined. Pathologists tend to avoid or ignore this controversial issue altogether and rely primarily on the readily identifiable dentate line as the anorectal border. However, by multidisciplinary consensus, the new edition of the staging manual defines the anal canal as beginning $1-2 \mathrm{~cm}$ above the dentate line. Therefore, tumors with an epicenter located $\leq 2 \mathrm{~cm}$ above the dentate line are staged as anal canal cancers.

\section{Tumor Size: For Some Things, Size Really Doesn't Matter}

Measurement on gross pathologic examination is considered the definitive determination of tumor size. Although it is recorded for purposes of documentation and may be important for quality control purposes (e.g., comparisons with dimensions derived via imaging modalities), tumor size is not related to outcome. Eight separate studies have shown that tumor size is of no prognostic significance in colorectal cancer $(4,5)$.

\section{Histologic Types: Some Matter, Some Don't}

For consistency and uniformity in pathologic reporting, the internationally accepted histologic classification proposed by the World Health Organization (Table $1 ; 6$ ) is recommended by the College of American Pathologists (7). It should be noted that medullary carcinoma was added to the revised World Health Organization classification published in 2000. Medullary carcinoma is a distinctive type of non-glandforming carcinoma that previously would have been classified as "undifferentiated carcinoma." It is composed of uniform polygonal tumor cells that exhibit solid growth in nested, organoid, or trabecular patterns and are characteristically infiltrated by lympho-

TABLE 1. World Health Organization Classification of Colorectal Carcinoma

Adenocarcinoma

Medullary carcinoma

Mucinous (colloid) adenocarcinoma ( $>50 \%$ mucinous)

Signet-ring cell carcinoma ( $>50 \%$ signet-ring cells)

Squamous cell (epidermoid) carcinoma

Adenosquamous carcinoma

Small-cell (oat cell) carcinoma

Undifferentiated carcinoma

Other (e.g., papillary carcinoma)

The term "carcinoma, NOS" (not otherwise specified) is not part of the WHO classification. 
cytes (tumor-infiltrating lymphocytes; 7). The importance of this unique type is its strong association with microsatellite instability, DNA repair gene dysfunction, and the hereditary nonpolyposis colon cancer syndrome (7-10).

For most histologic types of colorectal cancer, there is no stage-independent prognostic significance. The notable exceptions appear to be the rare types such as signet-ring cell carcinoma and smallcell carcinoma, which are prognostically unfavorable, and medullary carcinoma, which is prognostically favorable. However, the understanding of the prognostic value of histologic type is hampered by the paucity of data defining the relationship between histologic type, grade, and genetic characteristics of the tumor. This shortfall is particularly relevant to mucinous carcinoma, a histologic type representing a high proportion of microsatelliteunstable colorectal cancers but, overall, occurring most frequently without microsatellite instability. Thus, it is not surprising that among all of the histologic types of colonic cancer, the prognostic significance of mucinous carcinoma has been the most controversial.

A few studies, largely limited to univariate analyses, have indicated that mucinous adenocarcinoma may be an adverse prognostic factor $(4,5)$. More specifically, mucinous carcinoma has been linked with adverse outcome only if it occurs in specific anatomic regions of the bowel (e.g., the rectosigmoid) or in specific subsets of patients (e.g., those $<45$ years of age). In yet other studies, an association with decreased survival has been demonstrated only when mucinous carcinoma and signet-ring cell carcinoma have been grouped together and compared with typical adenocarcinoma. However, data of this type may be merely a reflection of the aggressive biologic behavior of signetring cell tumors. Only one multivariate analysis has shown mucinous carcinoma to be a stageindependent predictor of adverse outcome, but the study was limited to tumors presenting with large bowel obstruction, which is itself an adverse prognostic feature (11).

Signet-ring cell type of adenocarcinoma and small-cell (oat cell) carcinoma are the only histologic types of colonic carcinoma that consistently have been found to have a stage-independent adverse effect on prognosis. Small-cell carcinoma is a malignant neuroendocrine carcinoma that is similar histologically and biologically to small-cell (oat cell) carcinoma of the lung. Less clear is the general prognostic significance of focal neuroendocrine differentiation that may occur as a variable feature in other histologic types of colorectal cancer. Two studies, the most recent of which included a multivariate analysis of 350 cases, have indicated that extensive neuroendocrine differentiation may adversely affect outcome $(12,13)$.

In summary, based on current evidence, it must be concluded that the only histologic types of colorectal cancer that are prognostically significant are signet-ring cell and small-cell carcinomas (prognostically unfavorable) and medullary carcinoma (prognostically favorable). Mucinous carcinoma, when it is associated with microsatellite instability, is also prognostically favorable.

\section{Tumor Grade: Should We Be Grading "Pass/Fail"?}

In general practice, the histologic grading of colorectal cancer is largely subjective. Although a number of grading systems have been suggested in the literature, a single widely accepted and employed standard for grading is lacking. Among the suggested grading schemes, both the number of strata, as well as the criteria for distinguishing among them, vary markedly. In some systems, grade is defined on the basis of a single microscopic feature, such as the degree of gland formation, and in other systems, a large number of features are included in the evaluation (14). Irrespective of the complexity of the criteria, however, most systems stratify tumors into three or four grades as follows:

- Grade 1: Well differentiated

- Grade 2: Moderately differentiated

- Grade 3: Poorly differentiated

- Grade 4: Undifferentiated

Subjectivity and imprecision in grading may also be related in some degree to tumor heterogeneity. Intratumoral variation in the features used to assess grade may make the application of even the simplest grading system problematic. Thus, a significant degree of interobserver variability in colorectal cancer grading has been documented.

Despite these problems, histologic grade has been shown by numerous multivariate analyses to be a stage-independent prognostic factor in colorectal cancer $(4,5,7)$. Specifically, high tumor grade has been shown to be an adverse prognostic factor. In the vast majority of studies documenting the prognostic power of tumor grade, the subclassifications of the grading scheme have been collapsed to produce a two-tiered stratification for data analysis:

- Low Grade: Well differentiated and moderately differentiated

- High Grade: Poorly differentiated and undifferentiated

For the most part, the pathologic diagnosis of poorly differentiated or undifferentiated tumors is relatively consistent, and the associated interob- 
server variability is small. However, distinction between well- and moderately differentiated carcinomas is less reproducible and is associated with significant interobserver variability. A two-tiered grading system that eliminates this distinction might be expected to greatly improve diagnostic consistency. Given its proven prognostic value, relative simplicity, and reproducibility, a two-tiered grading system for colorectal carcinoma (i.e., low grade and high grade) has been recommended by a multidisciplinary colorectal working group of a consensus conference sponsored by the College of American Pathologists (4). In the proposed system, stratification is based solely on the proportion of gland formation by the tumor (e.g., greater than or less than $50 \%$ gland formation).

\section{Pathologic Stage}

\section{Pathologic versus Clinical Staging: The Power of " $p$ "}

The best estimation of prognosis in colorectal cancer is related to the anatomic extent of disease of disease determined on pathologic examination of the resection specimen. Although a large number of staging systems have been developed for colorectal cancer over the years, use of the TNM staging system of the AJCC and the International Union Against Cancer is widely recommended. Such use is encouraged by the College of American Pathologists, the Commission on Cancer of the American College of Surgeons, and the National Cancer Institute, as reflected in their recommended Common Data Elements for Colorectal to be used in all National Institutes of Health/National Cancer Institute-funded clinical research. The TNM system is also widely used by national, regional, and local tumor registries in the United States and Canada.

In the TNM system, the designation "T" refers to the local extent of the primary tumor at the time of diagnosis, before the administration of treatment of any kind (1). The designation "N" refers to the status of the regional lymph nodes, and " $M$ " refers to distant metastatic disease, including nonregional lymph nodes. The symbol "p," used as a prescript, refers to the pathologic determination of the TNM (e.g., pT1), as opposed to the clinical determination, designated by the prescript "c." Pathologic classification typically is based on gross and microscopic examination of the resection specimen of a previously untreated primary tumor. Assignment of pT requires a resection of the primary tumor or biopsy that is adequate to evaluate the highest $\mathrm{pT}$ category; $\mathrm{pN}$ entails removal of nodes adequate to validate lymph node metastasis; and $\mathrm{pM}$ implies microscopic examination of distant lesions. Clinical classification (cTMN) is usually determined by imaging techniques carried out before treatment dur- ing initial evaluation of the patient, or when pathologic classification is not possible. It is the grouping of a $\mathrm{T}$, an $\mathrm{N}$, and $\mathrm{M}$ parameter that determines the stage of the tumor and relates to prognosis. A TNM stage grouping can be constructed using a combination of clinically derived and pathologically derived data (e.g., pT1, cN0, cM0). However, when pathologic data become available (after surgical resection of the tumor, for example), they typically replace the corresponding clinically determined parameters. This convention is based on the assumption that pathologically derived data are more accurate.

The definitions of the individual TNM categories and stage groupings for colorectal carcinoma are shown in Table 2. TNM stage-related survival is shown in Table 3. It is considered the responsibility of the pathologist to assign a pTNM stage grouping when reporting on a colorectal cancer resection specimen $(4,7)$. Thus, the pathologically determined $\mathrm{T}$ and $\mathrm{N}$ categories of the tumor should be explicitly assigned and included in the pathology report. In most cases, distant metastatic disease is neither confirmed nor excluded by pathologic diagnosis, and in these circumstances, assignment of pMX (to document the lack of pathologically determined data) is appropriate.

\section{The Rules of the Game (the Old and the New)}

Specific issues related to the assignment of pathologic TNM are discussed in detail in this section.

Definition of pTis. For colorectal carcinomas, the staging category pTis (indicating carcinoma in situ) includes both malignant cells that are confined within the glandular basement membrane and those that invade the mucosal lamina propria, including those that extend up to but not through the muscularis mucosae. Penetration of the muscularis mucosae and invasion of the submucosa is classified as pT1.

It is noteworthy that for all organ systems other than the large intestine, "carcinoma in situ" refers exclusively to malignancy that has not yet penetrated the basement membrane of the epithelium from which it arose, and "invasive carcinoma" encompasses all tumors that penetrate the underlying stroma. Stromal invasion of any degree is a feature of extreme importance in all noncolorectal sites because of the possible access of tumor cells to stromal lymphatics or blood vessels and the consequent risk of metastasis. In colorectal cancer, however, the designation "pTis" (i.e., carcinoma in situ) is used to refer both to intraepithelial malignancies and to cancers that have invaded the mucosal stroma (intramucosal carcinomas), because the colonic mucosa is biologically unique. In contrast to the mucosa elsewhere in the gastrointestinal tract (or, indeed, in the entire body), tumor invasion of 


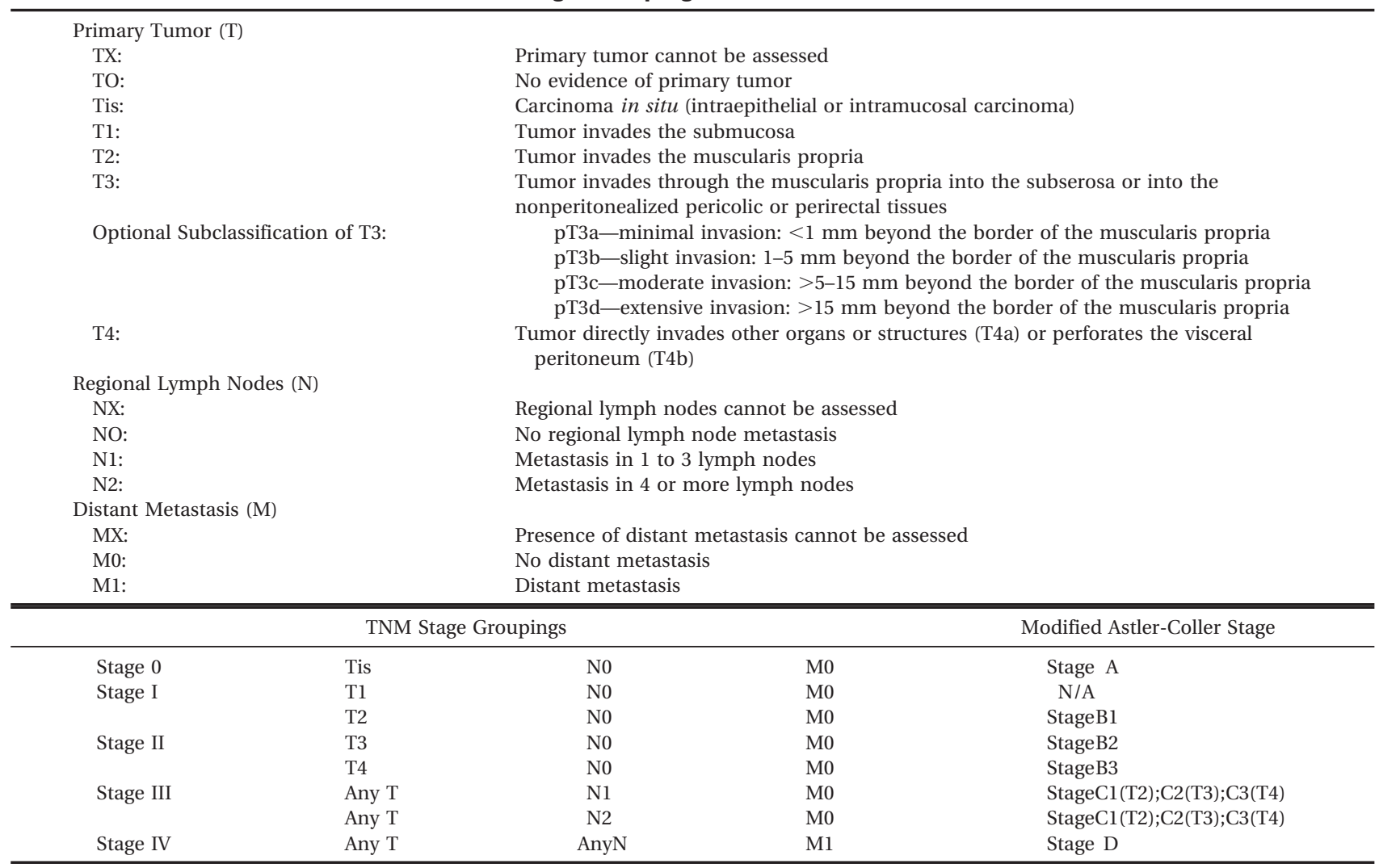

TABLE 3. Correlations between TNM Stage and Survival in Colorectal Carcinoma

\begin{tabular}{lc}
\hline \multicolumn{1}{c}{ TNM Stage } & 5-Year Survival (\%) \\
\hline Stage 0, I (Tis, T1; N0; M0) & $>90^{*}$ \\
Stage I (T2; N0; M0) & $80-85$ \\
Stage II (T3, T4; N0; M0) & $70-75$ \\
Stage III (T2; N1-3; M0) & $70-75$ \\
Stage III (T3; N1-3; M0) & $50-65$ \\
Stage III (T4; N1-3; M0) & $25-45$ \\
Stage IV (M1) & $<3$ \\
\hline
\end{tabular}

the lamina propria has no associated risk of regional nodal metastasis. Therefore, for the colon and rectum, inclusion of intramucosal carcinoma in the pTis category is justified. Nevertheless, the term "carcinoma in situ" in reference to colorectal cancer may be confusing, depending on whether it is used to refer to the T category of the TNM staging system or to intraepithelial tumor only, as it does in all other epithelial systems. Therefore, the terms "intraepithelial carcinoma" and "intramucosal carcinoma" are suggested descriptive terms for colorectal tumors in the pTis category $(4,5)$. High-grade (severe) dysplasia and intraepithelial carcinoma sometimes may be used synonymously, especially in cases of inflammatory bowel disease and in evaluation of index adenomas.

Subclassification of pT4. The highest category of local extent is pT4, which includes both penetration of an adjacent organ or structure (pT4a) and pen- etration of the parietal peritoneum, with or without involvement of an adjacent structure (pT4b). A free perforation of a colorectal carcinoma into the peritoneal cavity (i.e., a "hole" or transmural defect within the tumor) is also classified as T4b and is a dire prognostic factor. Unfortunately, in the TNM Manual, the word "perforation" is the descriptor used for T4b $(1,3)$ whether or not a "hole" is present, causing confusion as to the appropriate assignment of that category.

Pathologic diagnosis of serosal penetration by tumor is exceedingly important. A number of large studies have evaluated serosal penetration as a separate pathologic variable and have demonstrated by multivariate analysis that it has independent adverse prognostic significance. As shown in Table 4, the median survival time after surgical resection for cure is significantly shorter for pT4 tumors that penetrate the visceral peritoneum compared with the case of pT4 tumors without serosal involvement, with or without distant metastasis. A careful

TABLE 4. The Prognostic Significance of Serosal Involvement by Tumor in Colorectal Carcinoma

\begin{tabular}{lcc}
\hline & 5-Year Survival Rate (\%) & Median Survival Time (mo) \\
\hline pT4a, M0 & 49 & 58.2 \\
pT4b, M0 & 43 & 46.2 \\
pT4a, M1 & 12 & 22.7 \\
pT4b, M1 & 0 & 15.5 \\
\hline
\end{tabular}


pathologic study of local peritoneal involvement by Shepherd et al. (15) has suggested that the prognostic power of this feature alone may supersede that of either local extent of tumor (T category) or regional lymph node status ( $\mathrm{N}$ category).

Despite its biologic importance, serosal penetration is often underdiagnosed by pathologists. Documentation of peritoneal involvement by tumor demands meticulous pathologic analysis and may require extensive sampling and/or serial sectioning. Thus, it can be missed on routine histopathologic examination. In fact, it has been shown that cytologic examination of serosal scrapings reveals malignant cells in $26 \%$ of tumor specimens categorized as pT3 by histologic examination alone (16). In addition, the histopathologic findings associated with peritoneal penetration are heterogeneous, and standard guidelines for their diagnostic interpretation are lacking. These problems result in both substantial interobserver variation and underdiagnosis of peritoneal involvement because most pathologists tend to err on the side of conservative interpretation.

In the pathologic study by Shepherd et al. (15), the spectrum of microscopic features that may be seen with local peritoneal involvement by tumor was specifically addressed. Three types of local peritoneal involvement were defined:

1. a mesothelial inflammatory and/or hyperplastic reaction with tumor close to, but not at, the serosal surface

2. tumor present at the serosal surface with inflammatory reaction, mesothelial hyperplasia, and/or erosion/ulceration

3. free tumor cells on the serosal surface (in the peritoneum) with underlying ulceration of the visceral peritoneum

All three types of local peritoneal involvement can be used to define serosal involvement, and all are associated with shorter survival compared with tumor well clear of the serosal surface, especially the latter two. However, free cells on the serosal surface are more likely than the other forms of peritoneal involvement to predict intraperitoneal recurrence and/or persistence. It has been recommended that the definition of T4b be modified to encompass at least the latter two types of reactions outlined above $(4,7)$.

For T4a, it should be noted that direct invasion of other organs or structures includes invasion of other segments of the colorectum by way of the serosa or mesocolon (e.g., invasion of the sigmoid colon by carcinoma of the cecum). In contrast, intramural extension of tumor from one subsite (segment) of the large intestine into an adjacent subsite or into the ileum (e.g., for a cecal carcinoma) or anal canal (e.g., for a rectal carcinoma) does not affect the pT classification (17).
N Categories: The Magic Number of Nodes Needed. Stage-related outcome data are derived from studies in which the pathologic evaluation of the regional lymph nodes has been performed by conventional histologic staining of macroscopically identified lymph nodes. Because it has been shown that many nodal metastases in colorectal cancer are found in small lymph nodes $(<5 \mathrm{~mm}$ in diameter), diligent search for lymph nodes in resection specimens is essential (18). However, universally accepted standards for acceptable lymph node harvests and for handling of the recovered lymph nodes are lacking in general pathology practice.

Typically, all lymph nodes found are submitted either in part (e.g., half of a bisected node) or in toto for microscopic examination, and wide variation in the numbers of lymph nodes recovered from resection specimens exists. In truth, the actual number of lymph nodes present in any given resection specimen may be limited by anatomic variation, surgical technique, or both. However, it has been shown that a minimum of 12-15 lymph nodes must be examined to accurately predict regional node negativity (19-21). For this reason, it has been suggested that 12 lymph nodes be considered the minimum acceptable harvest from a careful specimen dissection and that if $<12$ nodes are found after careful gross examination, additional techniques (i.e., visual enhancement techniques such as fat clearing) be considered. It has been further recommended that all grossly negative or equivocal lymph nodes be submitted entirely for microscopic examination and that involvement of grossly positive lymph nodes be confirmed by either complete or partial microscopic examination.

Regional lymph nodes must be examined separately from lymph nodes outside of the anatomic site of the tumor because metastases in any lymph node in the regional nodal group are classified as $\mathrm{pN}$ disease, whereas all other nodal metastases are classified as pM1. The regional lymph node groups of the anatomic subsites of the colorectum are listed on Table 5. On microscopic examination, tumor in a regional lymph node, whether arriving there via afferent lymphatics or direct invasion through the capsule, is regarded as metastatic disease.

Micrometastasis and Extramural Nodules. Particularly problematic are discrete nodules of tumor found in the extramural adipose tissue on microscopic examination that may represent lymph nodes replaced by tumor but that, in the absence of residual nodal tissue, cannot be identified as nodal metastasis with certainty. To eliminate arbitrary decisions by pathologists as to whether or not such nodules are to be interpreted as nodal metastasis, the AJCC/International Union Against Cancer established guidelines for their interpretation that 
Cecum: anterior cecal, posterior cecal, ileocolic, right colic

Ascending colon: ileocolic, right colic, middle colic

Hepatic flexure: middle colic, right colic

Transverse colon: middle colic

Splenic flexure: middle colic, left colic, inferior mesenteric

Descending colon: left colic, inferior mesenteric, sigmoid

Sigmoid colon: inferior mesenteric, superior rectal sigmoidal, sigmoid mesenteric*

Rectosigmoid colon: perirectal*, left colic, sigmoid mesenteric, sigmoidal, inferior mesenteric, superior rectal, middle rectal

Rectum: perirectal*, sigmoid mesenteric, inferior mesenteric, lateral sacral, presacral, internal iliac, sacral promontory, superior rectal, middle rectal, inferior rectal

* Lymph nodes along the sigmoid arteries are considered pericolic nodes, and their involvement is classified as pN1 or pN2 according to the number involved. Perirectal lymph nodes include the mesorectal (paraproctal), lateral sacral, presacral, sacral promontory (Gerota), middle rectal (hemorrhoidal), and inferior rectal (hemorrhoidal) nodes. Metastasis in the external iliac or common iliac nodes is classified as pM1 (26).

were based on the size criteria. These guidelines were part of the version of the TNM staging system but were retired in January 2003, when the updated version of the TNM staging system went into effect. According to the old guideline, extramural tumor nodules within the regional lymph node distribution of the tumor that measured $>3 \mathrm{~mm}$ in diameter but lacked histologic evidence of residual lymph node tissue were classified as $\mathrm{pN}$ disease (1). Tumor nodules measuring $\leq 3 \mathrm{~mm}$ in diameter were classified in the pT3 category as discontinuous extramural extension of tumor. More recent data have suggested that extramural tumor deposits of any size correlate with decreased survival and do so independently of regional lymph node status. These data also have suggested that the number of pericolonic tumor deposits may be important as well, with increasing numbers being inversely proportional to disease-free survival. Thus, in the 6th edition of the AJCC Manual for Staging of Cancer, the "3-mm rule" was eliminated. According to the updated guideline, a discrete extramural tumor nodule with smooth contours, irrespective of size, is included in the $\mathrm{N}$ category as a positive lymph node (3).

The diagnosis of regional lymph node metastasis is limited to the use of conventional pathologic techniques (either gross or histologic). The biologic significance of minute amounts of metastatic tumor, known as "micrometastases," (tumor measuring $\leq 2.0 \mathrm{~mm}$ ), is controversial. Currently, the data are insufficient to recommend either the routine examination of multiple tissue levels of paraffin blocks or the use of special/ancillary techniques such as immunohistochemistry for epithelial and/or tumor-associated antigens (e.g., cytokeratin, carcinoembryonic antigen, etc.) or polymerase chain reaction (PCR) techniques to identify tumor RNA/DNA. All of these methods are costly, and some can be difficult to subject to quality control. More important, however, the significance of the findings generated from such analyses has yet to be proven. The use of these supplemental assays may result in upstaging of to $50 \%$ of tumors with histologically negative nodes, depending on the pT cat- egory $(22,23)$. However, micrometastasis has been shown to alter prognosis in some (24-29) but not all studies (29-34). Clinical validation will be required in well-designed studies before any of these methods are adopted for routine practice.

Pending definitive studies, it is recommended that any histologically identified focus of tumor that measures less than $2.0 \mathrm{~mm}$ but greater than $0.2 \mathrm{~mm}$ be classified as micrometastasis and assigned pN1 or pN2, as appropriate, by the pathologist. Cases with micrometastasis only should be designated $\mathrm{pN} 1(\mathrm{mi})$ or $\mathrm{pN} 2(\mathrm{mi})$. In contrast, cases with isolated tumor cells (ITC), single cells, or small clusters measuring $\leq 0.2 \mathrm{~mm}$ or ITC detected only by special studies such as immunohistochemistry or molecular techniques should be reported but should be classified as pNo.

M Categories: Documenting the Undocumented. As stated above, metastasis to any nonregional lymph node or metastasis to any distant organ or tissue is categorized as M1 disease. Peritoneal seeding of abdominal organs is also considered M1 disease, as is positive peritoneal fluid cytology. Isolated tumor cells found in the bone marrow are classified as distant micrometastasis, but, as with nodal micrometastasis (see above), their significance is as yet unproven.

Multiple tumor foci in the mucosa or submucosa of adjacent bowel (satellite lesions or skip metastasis) are not classified as distant metastasis (17). However, "satellite" lesions must be distinguished from additional primary tumors in which there is obvious evidence of origin from an overlying adenoma.

\section{The Rs: Residual Tumor and Recurrent Tumor and the $\mathrm{R}$ Classification}

By definition, the TNM categories describe the anatomic extent of malignant tumors that have not been previously treated, and the predictive value of the corresponding TNM stage groupings is based solely on data derived from outcome studies of such tumors after complete surgical resection. Tumor that remains in a resection specimen after 
previous (neoadjuvant) treatment of any type (radiation therapy alone, chemotherapy therapy alone, or any combined modality treatment) is codified by the TNM using a prescript " $y$ " to indicate the posttreatment status of the tumor (1). For many therapies, the classification of residual disease has been shown to be a strong predictor of posttreatment outcome. In addition, the ypTNM classification provides a standardized framework for the collection of data needed to accurately evaluate new therapies.

In contrast, tumor remaining in the patient after primary surgical resection (e.g., corresponding to a proximal, distal, or circumferential resection margin [see below] that is shown to be involved by tumor on pathologic examination) is categorized by a system known as $\mathrm{R}$ classification, which is as follows (35):

- RX: Presence of residual tumor cannot be assessed

- R0: No residual tumor

- R1: Microscopic residual tumor

- R2: Macroscopic residual tumor

In contrast to residual disease, tumor that is locally recurrent after a documented disease-free interval after surgical resection should be classified according to the TNM categories and modified with the prefix "r" (e.g., rpT1). By convention, the recurrent tumor is topographically assigned to the proximal segment of the anastomosis unless the proximal segment is small intestine $(1,17)$.

\section{The Matter of Margins}

The pertinent margins of a colorectal cancer resection specimen include the proximal, distal, and the mesenteric margins and, when appropriate, the circumferential (radial) margin (CRM). The CRM represents the retroperitoneal or perineal adventitial soft tissue margin closest to the deepest penetration of tumor. For all segments of the large intestine that are either incompletely encased (ascending colon, descending colon, upper rectum) or not encased (lower rectum) by peritoneum, the CRM is created by either sharp or blunt dissection of the retroperitoneal or subperitoneal aspect, respectively, at operation.

When the closest approach of tumor is $\geq 5 \mathrm{~cm}$ from the closest transverse (proximal or distal) margin, anastomotic recurrences are very rare. Therefore, it may be justified to forego histologic examination of the proximal and/or distal margin if they are $\geq 5 \mathrm{~cm}$ from the tumor (36). It has even been suggested (in guidelines from the Royal College of Pathologists in the United Kingdom) that donuts from stapling devices, which are the true margins of resection, need not be examined histologically of the tumor is $>3.0 \mathrm{~cm}$ from the cut end of the main specimen (37). In low anterior rectal resection specimens, however, distal margins are often critical because $5-\mathrm{cm}$ cuffs of normal mucosa may be hard to achieve. A margin of $2 \mathrm{~cm}$ of normal tissue is accepted as adequate to prevent local recurrence, and in many cases (T1 and T2 tumors), distal margins of $\leq 1 \mathrm{~cm}$ also prove sufficient (38).

An even more critical margin in rectal resection specimens is the CRM. Because of the cone-shaped anatomy of the lower rectum and its surrounding soft tissues, it is difficult to impossible to obtain a 2-cm cuff of marginal tissue circumferentially, especially posteriorly near the sacrococcygeal raffe. Not surprisingly, the CRM is the margin most frequently involved or most closely approached by tumor in rectal resection specimens. Multivariate analyses have suggested that tumor involvement of the CRM may be the single most critical factor in predicting local recurrence in rectal cancer (39-42). Moreover, in addition to predicting local recurrence, CRM involvement also has been shown to predict distant recurrence and overall survival. Unfortunately, in North America, the clinical importance of the CRM has not been widely recognized by pathologists, and routine pathologic evaluation of the CRM has been lacking. An assessment of data from three rectal cancer treatment protocols conducted by the North Central Cancer Treatment Group (NCCTG) between 1979 and 1992 showed that the CRM was evaluated pathologically in only $21 \%$ of cases $(43)$.

The CRM should be regarded as positive if the distance between the deepest extent of tumor and the closest CRM (i.e., the "surgical clearance" around the tumor) measures 0 to $1 \mathrm{~mm}$ on microscopic examination. Within this range, both local recurrence and overall survival rates are significantly elevated compared with clearance that is $>1$ $\mathrm{mm}$. In the NCCTG study, the local recurrence rate was $25 \%$ if the CRM clearance was $0-1 \mathrm{~mm}$ and was $3 \%$ if the clearance was $>1 \mathrm{~cm} \mathrm{(43)}$. Any malignant cells, whether present as direct extension of the primary tumor or as a lymph node metastasis, are relevant to the CRM assessment. Measurement of the surgical clearance in all rectal resection specimens, even those with negative CRMs, is also justified because local recurrence decreases and overall survival increases proportionately with increased surgical clearance. The overriding clinical relevance of tumor involvement of or proximity to the CRM mandates careful pathological evaluation and reporting of the microscopic status of the CRM for all rectal cancer resection specimens.

In prospective studies demonstrating significantly improved patient outcomes after surgeons have been trained in proper techniques for total mesorectal excision, the quality of the surgery has 
been judged in large part by the quality of the resection specimen. Pathological evaluation of the degree of intactness of the mesorectal surface has been shown to correlate clinically with both local recurrence and distant recurrence and pathologically with CRM involvement (44). Specifically, an incomplete mesorectum on gross pathological examination has been found to predict a higher rate of both CRM involvement and local recurrence, as compared with the case of a complete or nearly complete mesorectum. An incomplete mesorectum is defined as having little bulk and macroscopic defects down to the muscularis propria and/or a very irregular circumferential surface. In contrast, a complete or nearly complete mesorectum is recognized by full to moderate bulk and only minor to moderate irregularities of the mesorectal surface (Table 6; 44). Thus, the macroscopic quality of the rectal resection specimen, as judged by the amount of extramural soft tissue within the mesorectal envelope and the degree of intactness of the mesorectal fascia, directly reflects the adequacy of the surgical technique and correlates with clinically important predictors of outcome.

For segments of the colon that are completely encased by a peritonealized (serosal) surface (e.g., transverse and sigmoid colon), the mesenteric resection margin may be relevant as a radial margin because tumors may extend to this margin with (pT4) or without (pT3) penetration of the serosal surface. It should be examined when the point of deepest penetration of the tumor is on the mesenteric aspect of the colon, especially when the mesentery is trimmed close to the bowel wall. For those tumors limited to an antimesenteric peritonealized aspect of the bowel, the mesenteric margin is not relevant.

Because of its association with local recurrence, involvement of the radial or the mesenteric margin has implications for adjuvant therapy. Whether the primary tumor is classified as pT3 (without serosal penetration) or pT4b (with serosal penetration), resection is considered complete only if all surgical margins are negative, including the radial margin. That is, whether or not the tumor penetrates a serosal surface, resection is considered complete only if the resection margins are free of tumor. If a radial or mesenteric margin is involved by tumor, however, adjuvant therapy (e.g., local radiation) may be appropriate irrespective of the $\mathrm{T}$ category of the tumor.

\section{Bells and Whistles: Stage-Independent Prognostic Factors}

\section{Vessel Invasion: Venous versus Lymphatic}

In 10 different studies, venous invasion by tumor has been demonstrated by multivariate analysis to have an independent adverse impact on outcome; univariate analysis has also demonstrated this in several additional studies $(4,5,45)$. However, some studies identifying venous invasion as an adverse prognostic factor on univariate analysis have failed to confirm its independent impact on prognosis on multivariate analysis. Similarly disparate results have also been reported for lymphatic invasion (4, $5,45)$. In several studies, vascular invasion as a general feature was found to be prognostically significant by multivariate analysis, but no distinction between lymphatic and venous vessels was made. Yet in other studies, the location of the vascular involvement (e.g., invasion of extramural veins) has been a strong determinant of prognostic significance. Overall, therefore, data from existing studies are difficult to amalgamate. Nevertheless, the importance of venous and lymphatic invasion by tumor is strongly suggested and largely confirmed by the literature.

It is likely that the disparities among existing studies on vessel invasion are directly related to inherent problems related to the pathologic analysis of this feature. Definitive diagnosis of vessel invasion requires the identification of tumor within an endothelial-lined channel. However, assessment of vessel invasion may be difficult and may be complicated by tumor-induced fibrosis and fixation ar-

\section{TABLE 6. Macroscopic Pathologic Assessment of Total Mesorectal Excision Specimens*}

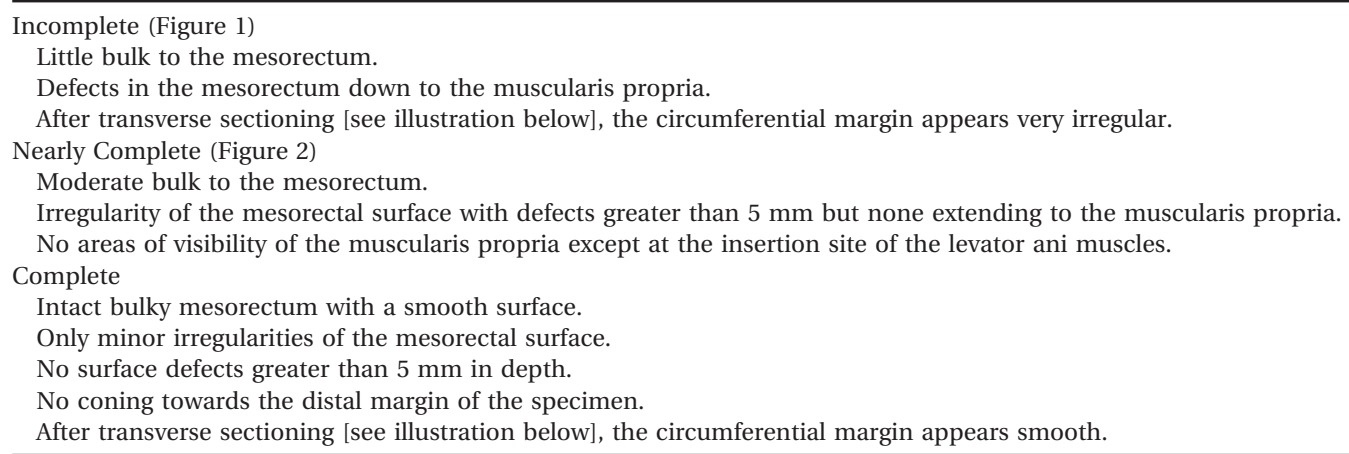

* The entire specimen is scored according to the worst area. 
tifact. Interobserver variability may be substantial in the interpretation of small-vessel (i.e., lymphatic or postcapillary venule) invasion, and large-vessel (i.e., muscular vein) invasion with tumor infiltration of the vessel wall and destruction of the vascular architecture may also be difficult to recognize. Special techniques such as immunohistochemical staining of endothelium or elastic tissue stains of venous walls may increase the ease and accuracy of evaluation. However, these techniques are labor intensive, time consuming, and expensive; therefore, they are not routinely performed. Additional limitations in the detection of vessel invasion are related to specimen sampling. For example, it has been shown that the reproducibility of detection of extramural venous invasion increases proportionally from $59 \%$, with examination of two blocks of tissue at the tumor periphery, to $96 \%$, with examination of five blocks (21). At present, however, no widely accepted standards or guidelines for the pathologic evaluation of vessel invasion exist, and pathology sampling practices may vary widely on both individual and institutional levels. Complicating this issue is the impact of cost containment on surgical pathology practice, which, in general, has tended to reduce overall sampling of resection specimens. The College of American Pathologists is recommending that at least three blocks (optimally, five blocks) of tumor at its point of deepest extent be submitted for microscopic examination (4).

\section{Tumor Border Configuration, Tumor Budding, and Perineural Invasion}

For colorectal cancer, the configuration of the tumor at the advancing edge (tumor border) has been shown to have prognostic significance that is independent of stage and may predict liver metastasis. Specifically, an irregular, infiltrating pattern of growth, as opposed to a smooth-pushing (expanding) border, has been demonstrated to be an independent adverse prognostic factor by several multivariate analyses. However, Jass et al. (46) showed that interobserver variability existed among pathologists evaluating tumor border configuration in general practice when no specific definitions or guidelines for assessment were provided. Agreement in diagnosis of infiltrating growth pattern was only $70 \%$ (fair). They also found that concordance improved to $90 \%$ when specific diagnostic criteria for defining infiltrating growth were employed (Table 7). It is significant that one of the microscopic criteria by which infiltrating growth can be recognized is perineural invasion. This pathologic feature is not routinely assessed or specifically reported in colorectal cancers, yet it has been shown by multivariate analysis in a number of studies to be an independent indicator of poor prognosis.

Tumor "budding" is another specific feature found at the tumor border that is variably ignored, reported separately, included in the overall assessment of tumor border configuration, or included in the overall assessment of tumor grade. It is defined as microscopic clusters of undifferentiated cancer cells just ahead of the invasive front of the tumor (47) and has also been referred to as "focal dedifferentiation." It may be seen in an otherwise wellor moderately differentiated tumor, and data from some studies have suggested that it is of greater prognostic value than overall grade $(14,47)$. It has been found to predict regional lymph node metastasis in APR specimens of $\mathrm{T} 1$ and superficial $\mathrm{T} 2$ rectal cancers, suggesting that it may be useful as an indicator for patients at risk of recurrence after local excision (transanal disk excision) alone (14). In the absence of definitive data on the biologic significance of tumor budding and of consensus on histologic grading in colorectal cancer, it would seem prudent to report this feature separately as a potentially important stage- and grade-independent prognostic factor.

\section{Host Lymphoid Response to Tumor}

Lymphocytic infiltration of tumor or peritumoral tissue is indicative of a host immunologic response to the invasive malignancy and has been shown by multivariate analysis in several studies to be a favorable prognostic factor $(4,5)$. In contrast, other studies either have failed to confirm the prognostic significance of a peritumoral lymphoid reaction or have demonstrated its significance only by univariate analysis. The results of these studies are difficult to compare because the histologic criteria for qualitative and quantitative evaluation differ from study to study. Some of the specific features that have been studied include perivascular lymphocytic cuffing in the muscularis propria, perivascular

\section{TABLE 7. Diagnostic Criteria for Infiltrating Tumor Border Configuration}

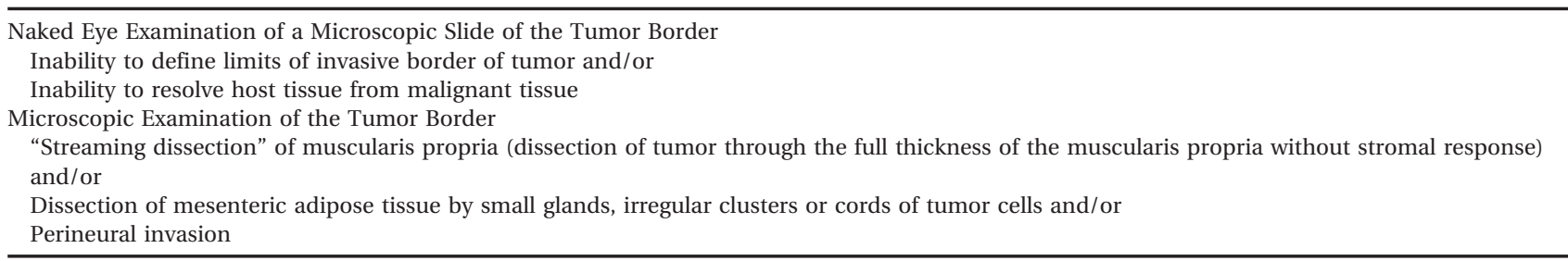


lymphocytic cuffing in the pericolonic fat or subserosa, lymphocytic infiltration at the tumor edge, and a transmural "Crohn's-like" lymphoid reaction (4). However, in some reports, little if any explanation of the criteria used for evaluation of this parameter has been offered. Therefore, although this feature appears promising as a favorable prognostic factor, further studies using comparable criteria are needed for confirmation.

Agreement has emerged, however, that large numbers of tumor-infiltrating lymphocytes (TILs) are uniquely associated with microsatellite instability in colorectal cancers (48) and, for that reason, may be a favorable prognostic factor. Indeed, large numbers of TILs are one of the diagnostic features of medullary carcinomas of the colorectum, but they may be found in other histologic types of tumors with microsatellite instability. Therefore it is recommended that TILs be distinguished from peritumoral lymphocytic infiltrates and that moderate to high densities of TILs (approximately four per high-power field) be reported (4).

\section{MOLECULAR PROGNOSTIC AND PREDICTIVE FACTORS: NOT-READY-FOR-PRIMETIME PLAYERS?}

Despite the prognostic power of stage, outcome for patients with tumors of like stage is heterogeneous. To more accurately individualize prognosis and plan appropriate adjuvant therapy, additional tissue-based prognostic indicators have been sought on a molecular level. A large number of molecular, protein, and carbohydrate markers have been investigated as possible prognostic factors, but none have yet been validated for patient care (4, 5). These include genotypic alterations such as c-myc amplification, her2/neu overexpression, or loss of heterozygosity at various chromosomal sites (e.g., $1 \mathrm{p}, 8 \mathrm{p}, 17 \mathrm{p}$, or $18 \mathrm{q}$ ), as well as markers of cell proliferation or angiogenesis, proteases or their receptors, and the expression of plasma membrane glycoproteins that may contribute to cell adhesion. Most of the studies on these putative prognostic markers are single-marker, retrospective, or small prospective investigations. Interpretation of these studies often has been hampered by poor quality clinical databases, inhomogeneity of adjuvant therapies, lack of standardization or quality control of the laboratory methodologies, or variability of statistical methodologies. Thus, none of these potential markers has been validated for patient care to date, but large prospective cooperative group studies are currently ongoing that will clarify the prognostic value of many of these factors. A partial list of putative prognostic markers in colorectal cancer taken from reports published in the past decade is shown in Table 8. Distinguishing themselves among these contenders are three candidates that will here be discussed in more detail.

\section{DCC/18q LOH: A Marker of Adverse Outcome}

Deleted in colon cancer (DCC) gene is a putative tumor suppressor gene that has been suggested to play an important role in cellular differentiation and epithelial stromal interaction. Most clinical studies on DCC have examined 18q LOH (loss of heterozygosity) by PCR amplification of polymorphic microsatellite markers at or near 18q21, and the results remain controversial. In retrospective studies, 18q LOH has been found to be predictive of shortened survival after adjustment for all other evaluated factors including tumor differentiation, vascular invasion, and TNM stage. In Stage II disease, 18q LOH has been found to predict shortened disease-free and overall survival in some studies $(49,50)$ but not others $(51-53)$. If validated as a predictor of adverse outcome in Stage II disease, diagnosis of DCC loss may be used in the future to identify patients for whom adjuvant chemotherapy is needed.

\section{MSI-H: A Marker of Favorable Outcome}

In colorectal cancer, altered DNA repair is characterized by nucleotide mismatch, also known as replication error (RER). Tumor RER status has been implicated as a predictor of outcome and treatment responsiveness in colorectal cancer. RER can be identified by alterations (instability) in inherited patterns of nucleotide repeats (microsatellites) scattered throughout the genome. Tumors display-

TABLE 8. Putative Prognostic Markers in Colorectal Cancer

$\begin{array}{ll}\text { CD44 v6 } & \text { Mn superoxide dismutase } \\ \text { C-reactive protein } & \text { Bc1-2, Bax } \\ \text { Hyaluronic acid } & \text { Thymidine phosphorylase } \\ \text { Si LeA } & \text { VEGF } \\ \text { Si LeX } & \text { Thymidylate synthase } \\ \text { CEA } & \text { Prolactin receptor } \\ \text { Sucrase-isomaltase } & \text { DCC/18q LOH } \\ \text { Plasminogen activator } & \text { P53/17p LOH } \\ \text { UPA receptor } & \text { P21 } \\ \text { Matrix metalloproteinases } & \text { P27 } \\ \text { Cathepsin B } & \text { C-myc } \\ \text { TIMP } & \text { HER2/neu }\end{array}$

Ki-ras

Vitamin D receptor

PCNA

NM23

Ki67

Cyclin D1

Ploidy

Chromosome 1 aneuploidy

Chromosome 11 aneuploidy

Microsatellite instability 
ing alterations in $\geq 30 \%$ of microsatellite markers tested are defined as MSI-H (microsatellite instability high). MSI-H status has been correlated with increased survival rates for tumors of like stage in some studies (53-58). Despite the correlation with favorable outcome, most studies have suggested that MSI-H tumors are less responsive to chemotherapy than are microsatellite-stable tumors.

\section{Thymidylate Synthase: a Marker of Chemosensitivity}

Thymidylate synthase (TS) is an essential enzyme needed for DNA synthesis in the S-phase of the cell cycle and is the target enzyme of 5-fluorouracil, the most important chemotherapeutic agent in the treatment of colorectal cancer. The clinical importance of TS protein has been suggested by studies demonstrating that intrinsic levels of TS correlate with resistance to 5-fluorouracil in experimental tumors and in the clinical setting. Preliminary data of intratumoral TS protein levels in patients with Stage II colon cancer have suggested that high TS protein expression is strongly associated with tumor recurrence, indicating that TS expression may be an independent prognostic factor in patients with colon cancer, independent of adjuvant treatment (58-61).

\section{SUMMARY: WHAT'S IMPORTANT NOW?}

The pathologic features of the resection specimen constitute the most powerful predictors of postoperative outcome in colorectal cancer. These include pathologic stage and stage-independent prognostic factors such as histologic grade, vascular invasion, perineural invasion, and tumor border features. Additional studies are needed to more precisely define guidelines for assessment of important prognostic factors and to eliminate variation in pathologic practice. Of unique importance in rectal cancer, the pathologic evaluation of the resection specimen serves as an objective indicator of the quality of completeness of total mesorectal excision and is itself predictive of outcome. No molecular markers have yet been validated for routine patient care.

\section{REFERENCES}

1. Fleming ID, Cooper JS, Henson DE, et al., editors. AJCC manual for staging of cancer. 5th ed. Philadelphia, PA: Lippincott-Raven; 1997.

2. Nelson H, Petrelli N, Carlin A, et al. Guidelines for colon and rectal cancer surgery. J Natl Cancer Inst 201;93:583-96.

3. Greene FL, Balch CM, Fleming ID, et al., editors. AJCC manual for staging of cancer. 6th ed. New York: Springer Verlag; 2002.

4. Compton CC, Fielding LP, Burgart LJ, et al. Prognostic factors in colorectal cancer: College of American Pathologists
Consensus Statement 1999. Arch Pathol Lab Med 2000;124: 979-94.

5. Compton CC, Fenoglio-Preiser CM, Pettigrew N, Fielding LP. American Joint Committee on Cancer Prognostic Factors consensus conference: Colorectal Working Group. Cancer 2000;88:1739-57.

6. Hamilton SR, Rubio CA, Vogelstein B, et al. Carcinoma of the colon and rectum. In: Hamilton SR, Aaltonen LA, editors. World Health Organization classification of tumours. Tumours of the digestive system. Lyon, France: IARC Press; 2000. p. 101-19.

7. Compton CC. Updated protocol for the examination of specimens removed from patients with colorectal carcinoma. Arch Pathol Lab Med 2000;124:1016-25.

8. Jass JR, Do K-A, Simms LA, Iino H, Wynter C, Pillay SP, et al. Morphology of sporadic colorectal cancer with DNA replication errors. Gut 1998;42:673-9.

9. Messerini L, Vitelli F, DeVitis LR, et al. Microsatellite instability in sporadic colorectal carcinomas: relationship to clinico-pathological variables. J Pathol 1997;182:380-4.

10. Jesserun J, Romero-Guadarrama M, Manivel JC. Medullary adenocarcinoma of the colon: clinicopathologic study of 11 cases. Hum Pathol 1999;30:843-8.

11. Mulcahy HE, Skelly MM, Husain A, et al. Long-term outcome following curative surgery for malignant large bowel obstruction. Br J Surg 1996;83:46-50.

12. DeBruine A, Wiggers T, Beek C, et al. Endocrine cells in colorectal adenocarcinomas: incidence, hormone profile and prognostic relevance. Int J Cancer 1993;54:765-71.

13. Gaffey M, Mills S, Lack E. Neuroendocrine carcinoma of the colon and rectum. A clinicopathologic, ultrastructural, and immunohistochemical study of 24 cases. Am J Surg Pathol 1990;14:1010-23.

14. Goldstein NS, Hart J. Histologic features associated with lymph node metastasis in stage $\mathrm{T} 1$ and superficial $\mathrm{T} 2$ rectal adenocarcinomas in abdominoperineal resections. Identifying a subset of patients for whom treatment with adjuvant therapy or completion abdominoperineal resection should be considered after local excision. Am J Clin Pathol 1999;111: 51-8.

15. Shepherd N, Baxter K, Love S. The prognostic importance of peritoneal involvement in colonic cancer: a prospective evaluation. Gastroenterology 1997;112:1096-102.

16. Zeng Z, Cohen AM, Hajdu S, et al. Serosal cytologic study to determine free mesothelial penetration of intraperitoneal colon cancer. Cancer 1992;70:737-40.

17. Wittekind C, Henson DE, Hutter RVP, Sobin LH, editors. TNM supplement. A commentary on uniform use. 2nd ed. New York: Wiley-Liss; 2001.

18. Herrera-Ornelas L, Justiniano J, Castillo N, et al. Metastases in small lymph nodes from colon cancer. Arch Surg 1987; 122:1253-6.

19. Scott KWM, Grace RH. Detection of lymph node metastases in colorectal carcinoma before and after fat clearance. Br J Surg 1989;76:1165-7.

20. Tepper JE, O'Connell MJ, Niedzwiecki D, et al. Impact of number of nodes retrieved on outcome in patients with rectal cancer. J Clin Oncol 2001;19:157-63.

21. Blenkinsopp WK, Stewart-Brown S, Blesovsky L, et al. Histopathology reporting in large bowel cancer. J Clin Pathol 1981;34:509-13.

22. Bernini A, Spencer M, Frizelle S, et al. Evidence for colorectal cancer micrometastases using reverse transcriptasepolymerase chain reaction analysis of MUC2 in lymph nodes. Cancer Detect Prev 2000;24:72-9.

23. Liefers GJ, Tollenaar RA, Cleton-Jansen AM. Molecular detection of minimal residual disease in colorectal and breast cancer. Histopathology 1999;34:385-90. 
24. Liefers GJ, Cleton-Jansen AM, van de Velde CJ, et al. Micrometastases and survival in stage II colorectal cancer. N Engl J Med 1998;339:223-8.

25. Yasuda K, Adachi Y, Shiraishi N, et al. Pattern of lymph node micrometastasis and prognosis of patients with colorectal cancer. Ann Surg Oncol 2001;8:300-4.

26. Clarke G, Ryan E, O'Keane JC, et al. The detection of cytokeratins in lymph nodes of Duke's B colorectal cancer subjects predicts a poor outcome. Eur J Gastroenterol Hepatol 2000;12:549-52.

27. Clarke G, Ryan E, Crowe J, et al. Immunohistochemical detection of mutant p53 protein in regional lymph nodes is associated with adverse outcome in stage II colorectal cancer. Eur J Histochem 1999;43:311-6.

28. Isaka N, Nozue M, Doy M, Fukao K. Prognostic significance of perirectal lymph node micrometastases in Dukes' B rectal carcinoma: an immunohistochemical study by CAM5.2. Clin Cancer Res 1999;5:2065-8.

29. Greenson JK, Isenhart CE, Rice $\mathrm{R}$, et al. Identification of occult micrometastases in pericolic lymph nodes of Duke's B colorectal cancer patients using monoclonal antibodies against cytokeratin and CC49. Correlation with long-term survival. Cancer 1994;73:563-9.

30. Clarke GA, Ryan E, Crowe JP, et al. Tumour-derived mutated K-ras. Int J Colorectal Dis 2001;16:108-11.

31. Tschmelitsch J, Klimstra DS, Cohen AM. Lymph node micrometastases do not predict relapse in stage II colon cancer. Ann Surg Oncol 2000;7:601-8.

32. Nakanishi Y, Ochiai A, Yamauchi Y, et al. Clinical implications of lymph node micrometastases in patients with colorectal cancers. A case control study. Oncology 1999;57:27680.

33. Oberg A, Stenling R, Tavelin B, Lindmark G. Are lymph node micrometastases of any clinical significance in Dukes Stages A and B colorectal cancer? Dis Colon Rectum 1998;41:1244-9.

34. Adell G, Boeryd B, Franlund B, et al. Occurrence and prognostic importance of micrometastases in regional lymph nodes in Dukes' B colorectal carcinoma: an immunohistochemical study. Eur J Surg 1996;162:637-42.

35. Wittekind C, Compton CC, Sobin LH. Residual tumour classification revisited. Cancer 2002;94:2511-6.

36. Cross SS, Bull AD, Smith JHF. Is there any justification for the routine examination of bowel resection margins in colorectal adenocarcinoma? J Clin Pathol 1989;42:1040-2.

37. Sloane JP, Ansell ID, Quirke P, Underwood JCE. Standards and minimum datasets for reporting common cancers. Minimum dataset for colorectal cancer histopathology reports. London: The Royal College of Pathologists; 1998.

38. Quirke P. Limitations of existing systems of staging for rectal cancer: the forgotten margin. In: Rajagopalan NT, editor. Rectal cancer research. New York: Springer-Verlag; 2001. p. 63-81.

39. Quirke P, Scott N. The pathologist's role in the assessment of local recurrence in rectal carcinoma. Surg Oncol Clin N Am 1992;3:1-17.

40. Quirke P, Durdy P, Dixon MF, Williams NS. Local recurrence of rectal adenocarcinoma due to inadequate surgical resection. Lancet 1986;II:996-9.

41. Adam IJ, Mohamdee MO, Martin IG, et al. Role of the circumferential margin involvement in the local recurrence of rectal cancer. Lancet 1994;344:707-11.

42. Chan K, Boey J, Wong S. A method of reporting radial invasion and surgical clearance of rectal carcinoma. Histopathology 1985;9:1319-27.
43. Stacchi L, Nelson H, Sargent DJ, et al. Impact of surgical and pathological variables in rectal cancer: a United States community and cooperative group report. J Clin Oncol 2001;19: 3895-902.

44. Nagtegaal ID, van der Worp E, van de Velde CJ, et al. Macroscopic evaluation of rectal cancer resection specimens: clinical significance of the pathologist in quality control. J Clin Oncol 2002;20:1729-34.

45. Compton CC. The pathology report in colon cancer: what's prognostically important? Dig Dis 1999;17:67-79.

46. Jass J, Atkin W, Cuzick J, et al. The grading of rectal cancer: historical perspectives and a multivariate analysis of 447 cases. Histopathology 1986;10:437-59.

47. Hase K, Shatney C, Johnson D, et al. Prognostic value of tumor "budding" in patients with colorectal cancer. Dis Colon Rectum 1993;36:627-35.

48. Jass JR, Do KA, Simms LA, et al. Morphology of sporadic colorectal cancer with DNA replication errors. Gut 1998;42: $673-9$.

49. Alexander J, Watanabe $\mathrm{T}, \mathrm{Wu} \mathrm{TT}$, et al. Histopathological identification of colon cancer with microsatellite instability. Am J Pathol 2001;158:527-35.

50. Jen J, Kim H, Piantadosi S, et al. Allelic loss of chromosome $18 \mathrm{q}$ and prognosis in colorectal cancer. N Engl J Med 1994; 331:213-21.

51. Laurent-Puig P, Olschwang S, Delattre O, et al. Survival and acquired genetic alterations in colorectal cancer. Gastroenterology 1992;102:1136-41.

52. Cohn KH, Ornstein DL, Wang F, et al. The significance of allelic deletions and aneuploidy in colorectal carcinoma. Cancer 1997;79:233-44.

53. Carethers JM, Hawn JK, Greenson JK, et al. Prognostic significance of allelic loss at chromosome 18q21 for stage II colorectal cancer. Gastroenterology 1998;114:1188-95.

54. Gryfe R, Kim H, Hsieh ET, et al. Tumor microsatellite instability and clinical outcome in young patients with colorectal cancer. N Engl J Med 2000;342:69-77.

55. Sankila R, Aaltonen LA, Jarvinen HJ, Mecklin JP. Better survival rates in patients with MLH1-associated hereditary colorectal cancer. Gastroenterology 1996;110:682-7.

56. Watanabe T, Wu TT, Catalano PJ, Ueki T. Molecular predictors of survival after adjuvant chemotherapy for colon cancer. N Engl J Med 2001;344:1196-206.

57. Guidoboni M, Gafa R, Viel A, Doglioni C. Microsatellite instability and high content of activated cytotoxic lymphocytes identify colon cancer patients with a favorable prognosis. Am J Pathol 2001;159:297-304.

58. Samowitz WS, Curtin K, Ma KN, Schaffer D. Microsatellite instability in sporadic colon cancer is associated with an improved prognosis at the population level. Cancer Epidemiol Biomarkers Prev 2001;10:917-23.

59. Berger SH, Jenh CH, Johnson LF, Berger FG. Thymidylate synthase overproduction and gene amplification in fluorodeoxyuridine-resistant human cells. Mol Pharmacol 1985;28:461-7.

60. Swain SM, Lippman ME, Egan EF, et al. Fluorouracil and high doses leucovorin in previously treated patients with metastatic breast cancer. J Clin Oncol 1989;7:890-9.

61. Leichman L, Lenz H-J, Leichman CG, et al. Quantitation of intratumoral thymidylate synthase expression predicts for resistance to protracted infusion of 5-fluorouracil and weekly leucovorin in disseminated colorectal cancers: preliminary report from an ongoing trial. Eur J Cancer 1995;31: 1306-10. 